\title{
A Search for Pulsed Very High-energy Gamma-Rays from 13 Young Pulsars in Archival VERITAS Data
}

A. Archer ${ }^{1}$, W. Benbow ${ }^{2}$ (D), R. Bird ${ }^{3}$ (iD), R. Brose ${ }^{4,5}$, M. Buchovecky ${ }^{3}$, J. H. Buckley ${ }^{1}$, A. J. Chromey ${ }^{6}$, W. Cui ${ }^{7,8}{ }^{(1 D}$, A. Falcone $^{9}$, Q. Feng ${ }^{10}$ (D) J. P. Finley ${ }^{7}$, L. Fortson ${ }^{11}$ (D), A. Furniss ${ }^{12}$ (D), A. Gent ${ }^{13}$, O. Gueta ${ }^{5}$, D. Hanna ${ }^{10}$ (D) T. Hassan ${ }^{5}$, O. Hervet ${ }^{14}$ (iD, J. Holder $^{15}$, G. Hughes ${ }^{2}$, T. B. Humensky ${ }^{16}$, C. A. Johnson ${ }^{14}$ (D), P. Kaaret ${ }^{17}$ (D), P. Kar ${ }^{18}$, N. Kelley-Hoskins ${ }^{5}$, M. Kertzman ${ }^{19}$, D. Kieda ${ }^{18}$ (D) F. Krennrich ${ }^{6}$, S. Kumar ${ }^{15}$, M. J. Lang ${ }^{20}$, T. T. Y. Lin ${ }^{10}$, A. McCann ${ }^{10}$, P. Moriarty ${ }^{20}$, R. Mukherjee ${ }^{21}$ (D), S. O’Brien ${ }^{22}$, R. A. Ong ${ }^{3}$, A. N. Otte ${ }^{13}$ (D), D. Pandel ${ }^{23}$, N. Park ${ }^{24}$, A. Petrashyk ${ }^{16}$, M. Pohl ${ }^{4,5}$ (D), E. Pueschel ${ }^{5}$ (D), J. Quinn ${ }^{22}$, K. $\operatorname{Ragan}^{10}$, G. T. Richards ${ }^{13,15}$ (D), E. Roache ${ }^{2}$, I. Sadeh ${ }^{5}$, M. Santander ${ }^{25}$ (D) S. S. Scott ${ }^{14}$, G. H. Sembroski ${ }^{7}$, K. Shahinyan ${ }^{11}$ (D), I. Sushch ${ }^{5}$ (D) J. Tyler ${ }^{10}$, S. P. Wakely ${ }^{24}$, A. Weinstein ${ }^{6}$, R. M. Wells ${ }^{6}$, P. Wilcox ${ }^{17}$, A. Wilhelm ${ }^{4,5}$, D. A. Williams ${ }^{14}$, T. J Williamson ${ }^{15}$, and B. Zitzer ${ }^{10}$

${ }^{1}$ Department of Physics, Washington University, St. Louis, MO 63130, USA

${ }^{2}$ Fred Lawrence Whipple Observatory, Harvard-Smithsonian Center for Astrophysics, Amado, AZ 85645, USA

${ }^{3}$ Department of Physics and Astronomy, University of California, Los Angeles, CA 90095, USA

${ }^{4}$ Institute of Physics and Astronomy, University of Potsdam, D-14476 Potsdam-Golm, Germany ${ }^{5}$ DESY, Platanenallee 6, D-15738 Zeuthen, Germany

${ }^{6}$ Department of Physics and Astronomy, Iowa State University, Ames, IA 50011, USA

${ }^{7}$ Department of Physics and Astronomy, Purdue University, West Lafayette, IN 47907, USA Department of Physics and Center for Astrophysics, Tsinghua University, Beijing 100084, People's Republic of China

${ }^{9}$ Department of Astronomy and Astrophysics, 525 Davey Lab, Pennsylvania State University, University Park, PA 16802, USA

${ }^{10}$ Physics Department, McGill University, Montreal, QC H3A 2T8, Canada; jonathan.tyler@mail.mcgill.ca

${ }^{11}$ School of Physics and Astronomy, University of Minnesota, Minneapolis, MN 55455, USA

${ }^{12}$ Department of Physics, California State University - East Bay, Hayward, CA 94542, USA

${ }^{13}$ School of Physics and Center for Relativistic Astrophysics, Georgia Institute of Technology, 837 State Street NW, Atlanta, GA 30332-0430, USA

grichard@udel.edu
${ }^{14}$ Santa Cruz Institute for Particle Physics and Department of Physics, University of California, Santa Cruz, CA 95064, USA

${ }^{15}$ Department of Physics and Astronomy and the Bartol Research Institute, University of Delaware, Newark, DE 19716, USA

${ }^{16}$ Physics Department, Columbia University, New York, NY 10027, USA

${ }^{17}$ Department of Physics and Astronomy, University of Iowa, Van Allen Hall, Iowa City, IA 52242, USA

${ }_{19}^{18}$ Department of Physics and Astronomy, University of Utah, Salt Lake City, UT 84112, USA

${ }^{19}$ Department of Physics and Astronomy, DePauw University, Greencastle, IN 46135-0037, USA

${ }^{20}$ School of Physics, National University of Ireland Galway, University Road, Galway, Ireland

${ }^{21}$ Department of Physics and Astronomy, Barnard College, Columbia University, NY 10027, USA

${ }^{2}$ School of Physics, University College Dublin, Belfield, Dublin 4, Ireland

${ }^{23}$ Department of Physics, Grand Valley State University, Allendale, MI 49401, USA

${ }^{24}$ Enrico Fermi Institute, University of Chicago, Chicago, IL 60637, USA

${ }^{25}$ Department of Physics and Astronomy, University of Alabama, Tuscaloosa, AL 35487, USA

Received 2018 August 27; revised 2019 March 28; accepted 2019 March 30; published 2019 May 8

\begin{abstract}
We conduct a search for periodic emission in the very high-energy (VHE) gamma-ray band $(E>100 \mathrm{GeV})$ from a total of 13 pulsars in an archival VERITAS data set with a total exposure of over $450 \mathrm{hr}$. The set of pulsars includes many of the brightest young gamma-ray pulsars visible in the Northern Hemisphere. The data analysis resulted in nondetections of pulsed VHE gamma-rays from each pulsar. Upper limits on a potential VHE gamma-ray flux are derived at the 95\% confidence level above three energy thresholds using two methods. These are the first such searches for pulsed VHE emission from each of the pulsars, and the obtained limits constrain a possible flux component manifesting at VHEs as is seen for the Crab pulsar.
\end{abstract}

Key words: gamma rays: general - pulsars: general - stars: neutron

Supporting material: figure set

\section{Introduction}

While just seven gamma-ray pulsars were known prior to the launch of the Fermi Large Area Telescope (LAT) in 2008, over 200 gamma-ray pulsars have now been discovered in the highenergy (HE) gamma-ray band above $100 \mathrm{MeV} .{ }^{26}$ However, in the gamma-ray band above $50 \mathrm{GeV}$ (as reported in the $2 \mathrm{FHL}$ catalog; see Ackermann et al. (2016)), only the Vela pulsar remains firmly detected in the LAT data. Further, in a stacked analysis of 115 gamma-ray pulsars by McCann (2015), no

\footnotetext{
${ }^{26}$ https://confluence.slac.stanford.edu/display/GLAMCOG/Public+List + of+LAT-Detected+Gamma-Ray+Pulsars
}

significant gamma-ray excess was observed at energies above $50 \mathrm{GeV}$. The shapes of the HE spectra for gamma-ray pulsars are well-characterized by exponential or subexponential cutoffs above energies of a few $\mathrm{GeV}$, where the fluxes are observed to fall rapidly. Natural cutoffs in the gamma-ray spectra at a few $\mathrm{GeV}$ are expected in synchro-curvature emission models, due to the radiation-reaction limit (Caraveo 2014). Synchro-curvature radiation from the outer magnetosphere remains the most accepted explanation found in the literature for the emission of the observed HE gamma-ray radiation, due to its ability to reproduce the general features of the observed pulsar light curves and spectra (Watters et al. 2009; Abdo et al. 2013; Pierbattista et al. 2015). 
Since the detection of the Crab pulsar in very high-energy (VHE) gamma-rays above $100 \mathrm{GeV}$ by VERITAS (Aliu et al. 2011) and MAGIC (Aleksić et al. 2012), one of the principal questions in VHE astrophysics has been whether or not the Crab pulsar is the sole VHE-emitting pulsar. The observed VHE emission from the Crab pulsar was found to be consistent with a pure power law extending from HE gamma-rays $>10 \mathrm{GeV}$ into the VHE band. These VHE measurements allowed stringent constraints to be placed on the location of the emission region and radiation mechanism responsible for the observed gamma-rays. Synchro-curvature radiation is not an adequate explanation for the observed radiation from the Crab pulsar into the VHE band, due to the absence of the expected spectral cutoff (Lyne \& Graham-Smith 2012). The VHE emission is generally accepted to be a result of inverseCompton (IC) scattering by some population(s) of relativistic electrons (e.g., Aharonian et al. 2012; Lyutikov et al. 2012; Harding \& Kalapotharakos 2015; Mochol \& Pétri 2015), though the proposed models largely diverge in their assumed emission locations and specifics of the IC scattering mechanism. Furthermore, the VHE spectrum of the Crab pulsar was measured to be consistent with a power law up to $1.5 \mathrm{TeV}$ by MAGIC (Ansoldi et al. 2016), with no evidence of a spectral cutoff. At present, it is not clear that any one model is capable of simultaneously reproducing the observed VHE light curve and energy spectrum of the Crab pulsar (Zanin 2017).

The Vela pulsar has recently been detected at energies above $50 \mathrm{GeV}$ by the Fermi-LAT (Leung et al. 2014) and up to $\sim 100 \mathrm{GeV}$ by H.E.S.S. II (Abdalla et al. 2018), making it the second pulsar detected from the ground by an imaging atmospheric Cherenkov telescope (IACT). The $10-100 \mathrm{GeV}$ spectrum seen by H.E.S.S. II is consistent with a pure power law, though the presence of curvature cannot yet be ruled out, given the current uncertainties, leaving open the question of the shape of the spectrum in this energy range.

Both the Crab and Vela pulsars belong to a population of young rotation-powered gamma-ray pulsars, a population that comprises over half of the total known gamma-ray pulsars. As two of the brightest young pulsars, the Crab and Vela are the most highly ranked ${ }^{27}$ among all known pulsars in terms of the detectability metric $\dot{E} / d^{2}$, where $\dot{E}$ is the spin-down luminosity and $d$ is the distance. However, the second-brightest gammaray pulsar (also of relatively high $\dot{E} / d^{2}$ ), Geminga, has been observed in deep exposures by VERITAS and MAGIC, and VHE pulsations were not detected in the searches conducted by either instrument (Aliu et al. 2015; Ahnen et al. 2016). Given that the Crab and Vela pulsars are the only ones known to emit at VHEs, a natural starting point for a search for more pulsars in the VHE band would be to selectively target based on $\dot{E} / d^{2}$, despite the nondetection of Geminga.

Many gamma-ray pulsars with the highest $\dot{E} / d^{2}$ are associated with known Galactic pulsar wind nebulae (PWNe), and PWNe are one of the dominant source classes detected at VHEs by IACT arrays. Through PWN searches, the major IACT observatories have each accumulated a large amount of data that can be probed for pulsed emission from the pulsar powering the nebula, regardless of whether the nebula is detected. Indeed, VERITAS has incidentally observed a number of pulsars while targeting a PWN or other object; 13 such pulsars are listed in Table 1, along with some of their

\footnotetext{
27 http://www.atnf.csiro.au/people/pulsar/psrcat/
}

properties. These 13 pulsars are hereafter referred to as the archival pulsars. The list contains eight of the top twelve pulsars in the Fermi-LAT second pulsar catalog (2PC) located in the northern sky visible to VERITAS when ranked in $\dot{E} / d^{2}$. Two of the top twelve are the Crab (rank 1) and Geminga (rank 5) pulsars, which have already been the subjects of VERITAS observational campaigns (Aliu et al. 2011, 2015) (though we note that the high ranking for Geminga is principally due to its small distance rather than a particularly high $\dot{E}$ ).

Brief synopses of some of the more notable pulsars investigated herein are given in the following.

1. PSR J0007+7303 is a radio-quiet pulsar (Halpern et al. 2004) associated with the supernova remnant CTA 1, which is detected in VHE gamma-rays above $500 \mathrm{GeV}$ (Aliu et al. 2013a). It is bright at GeV energies and has the second-highest spectral cutoff energy $(4.7 \mathrm{GeV})$ among all young gamma-ray pulsars in the 2PC (Abdo et al. 2013). Above the spectral cutoff energy, its HE gamma-ray spectrum is consistent with a subexponential cutoff ( $\mathrm{Li}$ et al. 2016).

2. PSR J0205+6449 is associated with 3C 58, which is a PWN detected at energies above $400 \mathrm{GeV}$ (Aleksić et al. 2014). It has the third-highest $\dot{E}$ of any gamma-ray pulsar in the 2PC (Abdo et al. 2013).

3. PSR J0357+3205 is the second slowest-rotating gammaray pulsar known (see footnote 26) and also one of the nearest known pulsars, at a distance of $\sim 0.5 \mathrm{kpc}$ (Marelli et al. 2013). It is notable for having a very long X-ray emission tail that extends $9^{\prime}$ behind the pulsar (De Luca et al. 2011). Its estimated runaway velocity of $1900 \mathrm{~km} \mathrm{~s}^{-1}$ makes it one of the highest-velocity pulsars known (Manchester et al. 2005).

4. PSR J2021+4026 is a radio-quiet gamma-ray pulsar (Abdo et al. 2009) located within the radio shell of the supernova remnant G78.2+2.1 (Ladouceur \& Pineault 2008). The remnant has also been detected as an extended source in the VHE band by VERITAS (Aliu et al. 2013b). The flux above $100 \mathrm{MeV}$ from the pulsar was seen to abruptly decrease by $\sim 20 \%$ within less than one week, which coincided with a decrease in the pulsar period time derivative, $\dot{P}$. After spending about three years in this lowflux, low $\dot{P}$ state, the HE flux and $\dot{P}$ gradually returned to their previous values over the course of a few months (Zhao et al. 2017). This is currently the only such observation of intermittent behavior (also called mode switching) seen for a pulsar at gamma-ray energies (Allafort et al. 2013). The sudden change in HE flux and $\dot{P}$ may be due to a change in the emission beaming from a shift in the magnetic field structure (Allafort et al. 2013).

5. PSR J2032+4127 is a pulsar that was thought to be isolated but was recently found to be in a long-period ( $P=45-50$ yr Ho et al. 2017) binary system (Lyne et al. 2015), orbiting a $15 M_{\odot}$ Be star (Hohle et al. 2010) companion. The pulsar is spatially coincident with the extended VHE gamma-ray source TeV J2032+4130, which would generally be interpreted as a PWN powered by the pulsar (Albert et al. 2008; Aliu et al. 2014). In recent observations conducted by VERITAS and MAGIC in fall 2017, both instruments observed a VHE flux elevated by a factor of $\sim 10$ over spring/summer 2017 (Mirzoyan \& Mukherjee 2017; Abeysekara et al. 2018). VERITAS and MAGIC additionally reported a 


\begin{tabular}{|c|c|c|c|c|c|c|c|c|c|c|}
\hline Pulsar & R.A. $\left({ }^{\circ}\right)$ & Decl. $\left({ }^{\circ}\right)$ & $P(\mathrm{~ms})$ & $\dot{P}\left(10^{-15}\right)$ & $\dot{E}\left(10^{34} \mathrm{erg} \mathrm{s}^{-1}\right)$ & Distance (kpc) & $\dot{E} / d^{2}$ Rank & Possible Counterpart & VERITAS Exposure (hr) & $\bar{\theta}_{\text {zenith }}\left({ }^{\circ}\right)$ \\
\hline $\mathrm{J} 0007+7303$ & 1.7565 & 73.0522 & 315.9 & 357 & 44.8 & $1.4 \pm 0.3$ (Pineault et al. 1993) & 9 & CTA 1 & 32.4 & 42 \\
\hline J0205+6449 & 31.4080 & 64.8286 & 65.7 & 190 & 2644 & $1.95 \pm 0.04(\mathrm{Xu}$ et al. 2006) & 3 & $3 \mathrm{C} 58$ & 22.2 & 35 \\
\hline $\mathrm{J} 0248+6021$ & 42.0776 & 60.3597 & 217.1 & 55.0 & 21.2 & $2.0 \pm 0.2($ Theureau et al. 2011$)$ & 12 & $\cdots$ & 45.9 & 32 \\
\hline J0357+3205 & 59.4680 & 32.0891 & 444.1 & 13.1 & 0.6 & $0.5_{-0.2}^{+0.4}$ (Marelli et al. 2013) & 14 & $\cdots$ & 7.92 & 14 \\
\hline $\mathrm{J} 0631+1036$ & 97.8657 & 10.6165 & 287.8 & 104 & 17.3 & $1.0 \pm 0.2($ Zepka et al. 1996) & 10 & $\cdots$ & 2.79 & 26 \\
\hline $\mathrm{J} 0633+0632$ & 98.4339 & 6.5418 & 297.4 & 79.6 & 11.9 & $<8.7$ & $\cdots$ & $\cdots$ & 108 & 29 \\
\hline $\mathrm{J} 1907+0602$ & 286.9782 & 6.0374 & 106.6 & 86.7 & 282 & $3.2 \pm 0.3$ (Abdo et al. 2010) & 8 & MGRO J1908+06 & 39.1 & 28 \\
\hline J1954+2836 & 298.5798 & 28.6013 & 92.7 & 21.2 & 105 & $<18.6$ & $\cdots$ & $\cdots$ & 5.18 & 16 \\
\hline J1958+2846 & 299.6667 & 28.7653 & 290.4 & 212 & 34.2 & $<18.5$ & $\cdots$ & $\cdots$ & 13.9 & 10 \\
\hline $\mathrm{J} 2021+3651$ & 305.2726 & 36.8513 & 103.7 & 95.6 & 338 & $1.8_{-1.4}^{+1.7}$ (Kirichenko et al. 2015) & 4 & Dragonfly Nebula & 58.2 & 18 \\
\hline J $2021+4026$ & 305.3781 & 40.4461 & 265.3 & 54.2 & 11.4 & $1.5 \pm 0.4$ (Landecker et al. 1980) & 13 & $\gamma$ Cygni & 20.6 & 21 \\
\hline $\mathrm{J} 2032+4127$ & 308.0548 & 41.4568 & 143.2 & 20.4 & 15 (Ho et al. 2017) & $1.44 \pm 0.05$ (Gaia Collaboration et al. 2018) & 11 & 47.9 & 21 & $\ldots$ \\
\hline $\mathrm{J} 2229+6114$ & 337.2720 & 61.2359 & 51.6 & 77.9 & 2231 & $0.80_{-0.20}^{+0.15}$ (Kothes et al. 2001) & 2 & Boomerang & 47.2 & 33 \\
\hline
\end{tabular}

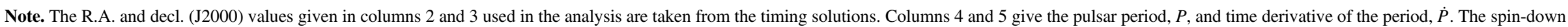

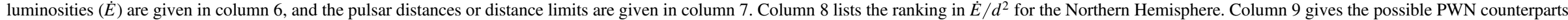

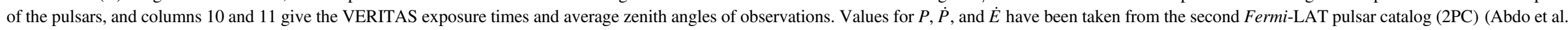

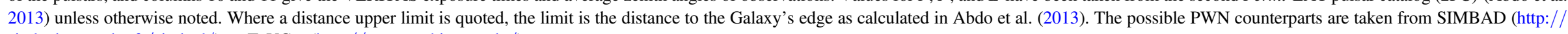
simbad.u-strasbg.fr/simbad/) or TeVCat (http://tevcat.uchicago.edu/). 

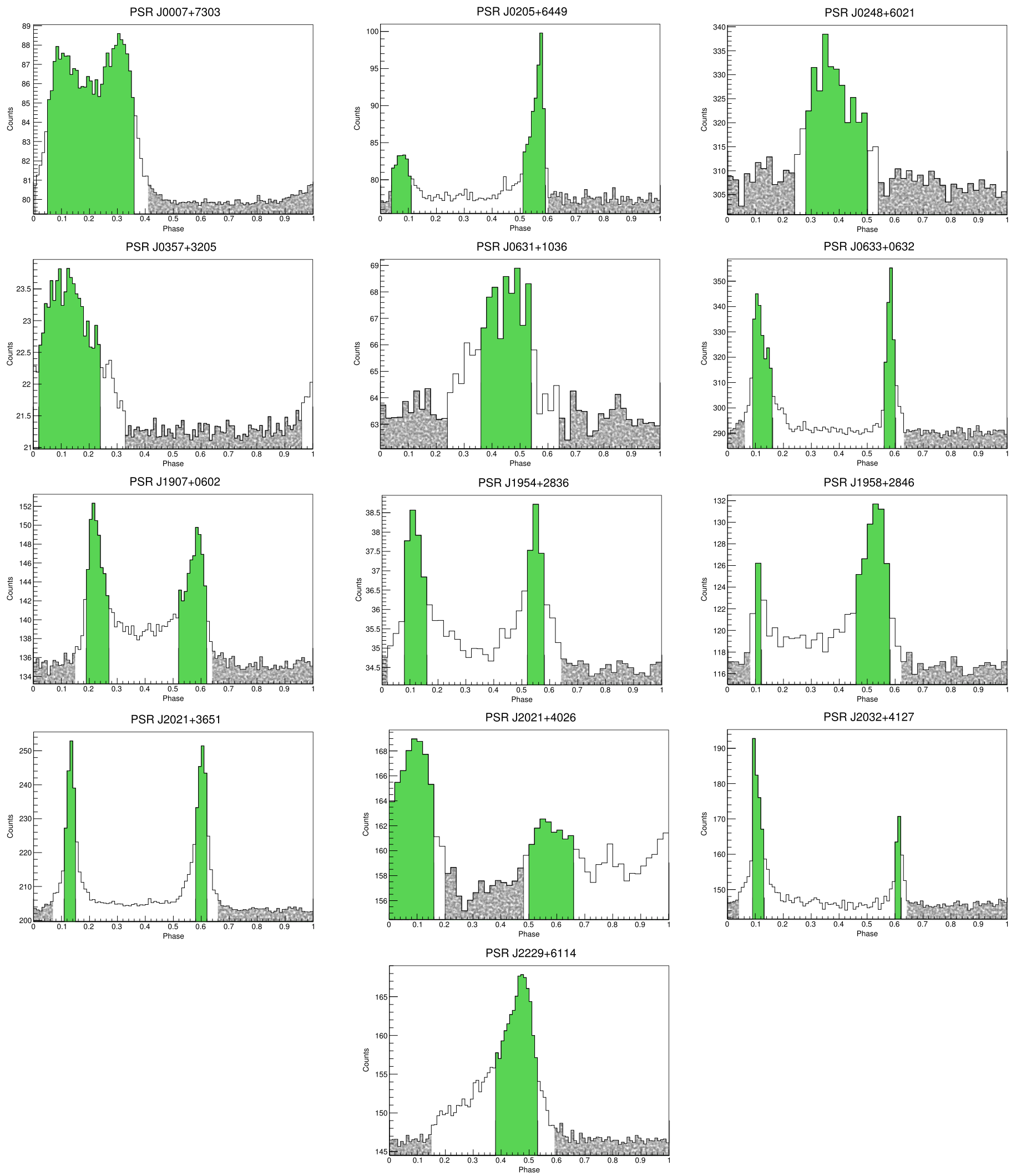

Figure 1. Light curves obtained from the phase-gating procedure (see text), showing the obtained phase-gate definitions for all 13 pulsars appearing in archival VERITAS data. The signal-counting regions, corresponding to the locations of P1 and P2 (where applicable), are shown in green, and the background-counting region is shown in gray (granulized).

clear detection of a point source at the pulsar location as the system neared periastron, confirming the presence of a gamma-ray binary.
Searches have been conducted for pulsed emission from all 13 of the pulsars appearing in archival VERITAS data-the first such VHE searches for each pulsar. The remainder of this 
Table 2

Phase-gate and Phase-offset Definitions

\begin{tabular}{lcccc}
\hline \hline Pulsar & P1 & P2 & Background & Phase Offset \\
\hline J0007+7303 & $0.05-0.36$ & None & $0.41-0.01$ & -0.0725 \\
J0205+6449 & $0.04-0.11$ & $0.51-0.59$ & $0.60-0.04$ & -0.1455 \\
J0248+6021 & $0.28-0.50$ & None & $0.54-0.24$ & 0.033 \\
J0357+3205 & $0.02-0.24$ & None & $0.33-0.96$ & 0.003 \\
J0631+1036 & $0.36-0.54$ & None & $0.64-0.24$ & 0.023 \\
J0633+0632 & $0.56-0.60$ & $0.09-0.16$ & $0.63-0.06$ & 0.0145 \\
J1907+0602 & $0.52-0.62$ & $0.19-0.27$ & $0.64-0.15$ & 0.002 \\
J1954+2836 & $0.52-0.58$ & $0.08-0.16$ & $0.64-0.02$ & 0.0125 \\
J1958+2846 & $0.46-0.58$ & $0.10-0.12$ & $0.62-0.08$ & 0.0135 \\
J2021+3651 & $0.58-0.62$ & $0.11-0.15$ & $0.66-0.07$ & 0.0355 \\
J2021+4026 & $0.00-0.16$ & $0.50-0.66$ & $0.20-0.48$ & -0.0495 \\
J2032+4127 & $0.60-0.62$ & $0.09-0.13$ & $0.64-0.04$ & 0.1585 \\
J2229+6114 & $0.38-0.53$ & None & $0.59-0.15$ & -0.0635 \\
\hline
\end{tabular}

Note. Columns 2 and 3 give the gate definitions for the first peak P1 and the second peak P2 (if present), respectively. Column 4 gives the background phase-gate definitions. Column 5 lists the phase offsets between the timing solutions used and those appearing in the $2 \mathrm{PC}$, which were used to calculate the phase gates.

article is structured in the following way: in Section 2, the Fermi-LAT and VERITAS data selection and analysis methods are summarized; Section 3 gives the results of the searches for VHE pulsed emission; and in Section 4, we discuss these results and offer concluding remarks.

\section{Data Selection and Analysis}

\subsection{VERITAS}

VERITAS is an array of four $12 \mathrm{~m}$ diameter IACTs located at the Fred Lawrence Whipple Observatory in Southern Arizona $\left(31^{\circ} 40^{\prime} \mathrm{N}, 110^{\circ} 57^{\prime} \mathrm{W}, 1.3 \mathrm{~km}\right.$ a.s.l.). Full-array operations started in Spring 2007. The telescopes are a Davies-Cotton design with reflectors consisting of 345 adjustable hexagonal mirror facets and cameras comprising 499 photomultiplier tubes covering a field of view (FoV) of $\sim 3.5$. VERITAS is sensitive to $\mathrm{VHE}$ gamma-ray photons in the energy range $85 \mathrm{GeV}$ to $>30 \mathrm{TeV}$, with sufficient sensitivity to detect a $1 \%$ Crab Nebula source in approximately $25 \mathrm{hr}$. It has an angular resolution of $\sim 0{ }^{\circ} 1$ at $68 \%$ containment averaged over the VERITAS energy range, and a pointing-accuracy error of less than 50 arcsec (Park \& VERITAS Collaboration 2015). The VERITAS data analysis results presented herein are obtained using the general methodology outlined in Acciari et al. (2008).

Given that the analysis for each pulsar is performed on archival VERITAS data, the amount of available data for each pulsar varies considerably. The location of each pulsar is taken to be that provided in the corresponding pulsar timing solution, and these coordinates are given in Table 1. The data selected for analysis satisfy two criteria: (a) an archival pulsar is within 1.5 of the center of the instrument FoV, and (b) the date on which the data were taken falls within the epoch of validity of the corresponding timing solution used to phase fold the photon arrival times. The timing solutions used for the analyses presented here were obtained from the LAT Gamma-Ray Pulsar Timing Models public webpage (see footnote 26). The data undergo a quality-selection process, with the sum of all VERITAS data analyzed here constituting a total exposure time of $451.3 \mathrm{hr}$. The exposure time for each individual pulsar is given in column 2 of Table 3 . After the VERITAS data are processed through the standard analysis pipeline, the data are phase-folded with the appropriate timing solution, using the Tempo2 pulsar timing software package (Hobbs et al. 2006).

A total of six periodicity tests are applied to the phase-folded data for each pulsar. All three sets of the standard VERITAS data selection cuts are used for each individual analysis, where each set was originally generated in such a way as to optimize sensitivity for power-law spectral shapes varying from soft to hard. The sets of cuts applied to the data are hereafter referred to as soft, moderate, or hard. The primary effect of applying these three sets of cuts is establishing three different energy thresholds per analysis, which is done in order to minimize a priori assumptions about the energy above which emission may be seen. The application of soft cuts gives the lowest analysis energy threshold of the three sets, which therefore provides the most sensitivity for potential "Crab pulsar-like" power-law spectral shapes extending from the HE band into the VERITAS energy range, while moderate and hard cuts are better suited for searching for possible additional spectral components manifesting at higher energies in the VHE band, due to the higher thresholds. For each set of cuts, two independent tests for a pulsed signal in the phase-folded VERITAS data are conducted. The first test uses the a priori-defined expected signal and background phase regions (henceforth referred to as the "phasegate test") described in Section 2.3. The significance is calculated, using Equation (17) in Li \& Ma (1983), from the unbinned phase data by counting $N_{\text {on }}$ and $N_{\text {off }}$ in the gated phase regions and determining the ratio of the sizes of the signal- and background-counting regions, $\alpha$. For the second test, de Jager's $H$ test (de Jager et al. 1989) is applied to the unbinned phase data; the advantage of this test is that it requires no a priori knowledge of expected pulse location(s) in the light curves. The total number of tests is, therefore, six per pulsar search.

\subsection{Fermi-LAT}

The Fermi LAT is an electron-positron pair-conversion telescope sensitive to gamma-ray photons with energies between $\sim 20 \mathrm{MeV}$ and $>300 \mathrm{GeV}$. It has a FoV of $\sim 2.5 \mathrm{sr}$ and attains full-sky coverage approximately every three hours. For a complete description of the instrument, see Atwood et al. (2009) and Ackermann et al. (2012).

A total of $7.6 \mathrm{yr}$ of Fermi-LAT data for each pulsar is analyzed. To generate Fermi-LAT spectra for each of the archival pulsars, the Fermi-LAT Science Tools (version v10r0p5) are used with the standard quality cuts for a Galactic point-source analysis, as detailed on the public LAT Data Selection Recommendations webpage. $^{28,29}$ Events with energies between $100 \mathrm{MeV}$ and $300 \mathrm{GeV}$ collected within a $20^{\circ}$ region-of-interest (ROI) of the 3FGL catalog (Acero et al. 2015) location of each pulsar are processed with the maximum-likelihood fitting routine using the Pass 8 instrument response functions.

The spectral reconstruction methodology used here follows the same steps outlined in the second Fermi-LAT pulsar catalog (Abdo et al. 2013). To generate spectral energy distributions of the Fermi-LAT data for each pulsar, a maximum likelihood analysis is performed in each of 12 logarithmically spaced energy bins spanning the range of $100 \mathrm{MeV}-300 \mathrm{GeV}$. Spectral models for all sources in the 3FGL catalog in the ROI in addition to the

\footnotetext{
28 https://fermi.gsfc.nasa.gov/ssc/data/analysis/documentation/Cicerone/ Cicerone_Data_Exploration/Data_preparation.html

29 Maximum zenith angle $=90^{\circ}$; event class $=128$; IRF name $=$ P8R2_SOURCE_V6.
} 
Table 3

Results for the 13 Pulsars Appearing in Archival VERITAS Data

\begin{tabular}{|c|c|c|c|c|c|c|c|}
\hline Pulsar & $\begin{array}{l}\text { Exposure } \\
\text { Time (hr) }\end{array}$ & Cut Type & Significance & $H$ Statistic & $\begin{array}{c}\text { Spectral Analysis Thresh- } \\
\text { old }(\mathrm{GeV})\end{array}$ & $\begin{array}{c}\begin{array}{c}H \text {-Test Flux UL }\left(10^{-9}\right. \\
\left.\mathrm{m}^{-2} \mathrm{~s}^{-1}\right)\end{array}\end{array}$ & $\begin{array}{c}\text { Rolke Flux UL }\left(10^{-9}\right. \\
\left.\mathrm{m}^{-2} \mathrm{~s}^{-1}\right)\end{array}$ \\
\hline $\mathrm{J} 0007+7303$ & 32.4 & $\begin{array}{c}\text { soft } \\
\text { moderate } \\
\text { hard }\end{array}$ & $\begin{array}{l}-1.74 \\
-0.95 \\
-0.51\end{array}$ & $\begin{array}{l}4.32 \\
2.37 \\
3.15\end{array}$ & $\begin{array}{r}320 \\
460 \\
1100\end{array}$ & $\begin{array}{c}16.7 \\
6.20 \\
1.38\end{array}$ & $\begin{array}{l}1.24 \\
2.48 \\
0.767\end{array}$ \\
\hline J0205+6449 & 22.2 & $\begin{array}{c}\text { soft } \\
\text { moderate } \\
\text { hard }\end{array}$ & $\begin{array}{l}-1.29 \\
-1.11 \\
-1.40\end{array}$ & $\begin{array}{l}1.28 \\
3.29 \\
3.94\end{array}$ & $\begin{array}{l}240 \\
350 \\
500\end{array}$ & $\begin{array}{c}13.7 \\
7.63 \\
4.12\end{array}$ & $\begin{array}{l}2.77 \\
1.63 \\
0.575\end{array}$ \\
\hline $\mathrm{J} 0248+6021$ & 45.9 & $\begin{array}{c}\text { soft } \\
\text { moderate } \\
\text { hard }\end{array}$ & $\begin{array}{l}0.00 \\
0.85 \\
0.44\end{array}$ & $\begin{array}{l}3.26 \\
3.69 \\
1.34\end{array}$ & $\begin{array}{l}220 \\
290 \\
600\end{array}$ & $\begin{array}{r}19.4 \\
10.7 \\
1.9\end{array}$ & $\begin{array}{c}11.0 \\
8.65 \\
1.72\end{array}$ \\
\hline $\mathrm{J} 0357+3205$ & 7.92 & $\begin{array}{c}\text { soft } \\
\text { moderate } \\
\text { hard }\end{array}$ & $\begin{array}{r}-0.47 \\
-0.17 \\
0.12\end{array}$ & $\begin{array}{l}0.74 \\
0.32 \\
2.36\end{array}$ & $\begin{array}{l}140 \\
200 \\
380\end{array}$ & $\begin{array}{c}33.6 \\
10.7 \\
5.26\end{array}$ & $\begin{array}{c}20.9 \\
10.1 \\
4.01\end{array}$ \\
\hline $\mathrm{J} 0631+1036$ & 2.79 & $\begin{array}{c}\text { soft } \\
\text { moderate } \\
\text { hard }\end{array}$ & $\begin{array}{r}-1.27 \\
0.81 \\
-1.07\end{array}$ & $\begin{array}{l}3.61 \\
0.56 \\
1.44\end{array}$ & $\begin{array}{l}150 \\
220 \\
460\end{array}$ & $\begin{array}{c}79.4 \\
18.2 \\
7.44\end{array}$ & $\begin{array}{c}13.9 \\
22.2 \\
2.44\end{array}$ \\
\hline J0633+0632 & 108 & $\begin{array}{c}\text { soft } \\
\text { moderate } \\
\text { hard }\end{array}$ & $\begin{array}{r}-1.37 \\
0.41 \\
0.70\end{array}$ & $\begin{array}{l}3.66 \\
0.32 \\
4.80\end{array}$ & $\begin{array}{l}180 \\
260 \\
500\end{array}$ & $\begin{array}{l}8.92 \\
1.95 \\
1.01\end{array}$ & $\begin{array}{l}1.00 \\
1.59 \\
0.523\end{array}$ \\
\hline J1907+0602 & 39.1 & $\begin{array}{c}\text { soft } \\
\text { moderate } \\
\text { hard }\end{array}$ & $\begin{array}{r}-1.49 \\
0.36 \\
-0.15\end{array}$ & $\begin{array}{c}1.60 \\
10.4 \\
2.60\end{array}$ & $\begin{array}{l}180 \\
260 \\
550\end{array}$ & $\begin{array}{c}11.7 \\
7.72 \\
1.73\end{array}$ & $\begin{array}{l}1.72 \\
3.72 \\
0.953\end{array}$ \\
\hline J1954+2836 & 5.18 & $\begin{array}{c}\text { soft } \\
\text { moderate } \\
\text { hard }\end{array}$ & $\begin{array}{r}1.07 \\
0.58 \\
-1.50\end{array}$ & $\begin{array}{l}7.01 \\
2.46 \\
0.60\end{array}$ & $\begin{array}{l}130 \\
200 \\
290\end{array}$ & $\begin{array}{c}68.4 \\
19.3 \\
8.24\end{array}$ & $\begin{array}{c}40.3 \\
14.0 \\
1.48\end{array}$ \\
\hline J1958+2846 & 13.9 & $\begin{array}{c}\text { soft } \\
\text { moderate } \\
\text { hard }\end{array}$ & $\begin{array}{l}-0.70 \\
-1.24 \\
-1.54\end{array}$ & $\begin{array}{l}1.62 \\
0.82 \\
3.00\end{array}$ & $\begin{array}{l}130 \\
180 \\
260\end{array}$ & $\begin{array}{c}24.9 \\
9.49 \\
6.81\end{array}$ & $\begin{array}{l}8.62 \\
2.24 \\
0.658\end{array}$ \\
\hline $\mathrm{J} 2021+3651$ & 58.2 & $\begin{array}{c}\text { soft } \\
\text { moderate } \\
\text { hard }\end{array}$ & $\begin{array}{r}-0.56 \\
0.25 \\
0.95\end{array}$ & $\begin{array}{l}9.46 \\
2.28 \\
6.46\end{array}$ & $\begin{array}{l}150 \\
220 \\
420\end{array}$ & $\begin{array}{c}25.4 \\
7.23 \\
2.48\end{array}$ & $\begin{array}{l}4.53 \\
2.96 \\
1.06\end{array}$ \\
\hline $\mathrm{J} 2021+4026$ & 20.6 & $\begin{array}{c}\text { soft } \\
\text { moderate } \\
\text { hard }\end{array}$ & $\begin{array}{r}0.18 \\
0.15 \\
-1.93\end{array}$ & $\begin{array}{l}0.73 \\
3.28 \\
2.42\end{array}$ & $\begin{array}{l}170 \\
240 \\
460\end{array}$ & $\begin{array}{c}24.1 \\
15.0 \\
4.68\end{array}$ & $\begin{array}{l}32.1 \\
13.8 \\
0.0615\end{array}$ \\
\hline $\mathrm{J} 2032+4127$ & 47.9 & $\begin{array}{c}\text { soft } \\
\text { moderate } \\
\text { hard }\end{array}$ & $\begin{array}{r}-0.37 \\
0.58 \\
0.42\end{array}$ & $\begin{array}{l}0.34 \\
4.29 \\
2.00\end{array}$ & $\begin{array}{l}170 \\
220 \\
460\end{array}$ & $\begin{array}{c}10.9 \\
10.4 \\
2.22\end{array}$ & $\begin{array}{l}4.07 \\
3.56 \\
0.974\end{array}$ \\
\hline $\mathrm{J} 2229+6114$ & 47.2 & $\begin{array}{c}\text { soft } \\
\text { moderate } \\
\text { hard }\end{array}$ & $\begin{array}{r}0.72 \\
0.19 \\
-0.75\end{array}$ & $\begin{array}{l}0.30 \\
0.58 \\
2.35\end{array}$ & $\begin{array}{l}240 \\
320 \\
660\end{array}$ & $\begin{array}{l}8.75 \\
5.28 \\
1.97\end{array}$ & $\begin{array}{l}9.41 \\
4.07 \\
0.648\end{array}$ \\
\hline
\end{tabular}

Note. Each pulsar has three sets of results, one for each set of cuts applied to the data. Column 2 lists the exposure time for each pulsar, copied here from Table 1 for convenience. Column 3 specifies the set of cuts used in the analysis. Columns 4 and 5 give the phase-gate test pre-trials significance and $H$ statistic, respectively. Column 6 gives the spectral analysis energy threshold in GeV. Integral flux upper limits at the 95\% CL above the spectral analysis threshold in column 6 from the $H$ test and Rolke methods are given in columns 7 and 8, respectively.

galactic and isotropic diffuse backgrounds (gll_iem_v06.fits, iso_p8r2_source_V6_v06.txt) are included in the likelihood fitting. ${ }^{30}$ The normalization parameters of the galactic and

\footnotetext{
${ }^{30}$ We note that the 3FGL model files are based on an analysis of four years of LAT data, while here we use $7.6 \mathrm{yr}$ of data. To assess the impact of new sources that could be detectable in a roughly doubled data set, we reprocessed all of the LAT data with model files derived from the Preliminary LAT $8 \mathrm{yr}$ Point Source List (FL8Y). We find that the reconstructed LAT spectra using the FL8Y model files are the same, within errors, as those presented in this study in Section 3.
}

isotropic diffuse models and all sources within a circle of $4^{\circ}$ in radius centered at the pulsar location are left free in the fitting routine, while all other sources have parameters fixed to their 3FGL values. For the computation of the spectral points, in each energy bin the pulsar is modeled as a point source with a simple power-law spectral shape:

$$
\frac{d F}{d E}=\boldsymbol{F}_{0}\left(\frac{E}{E_{0}}\right)^{-\gamma},
$$




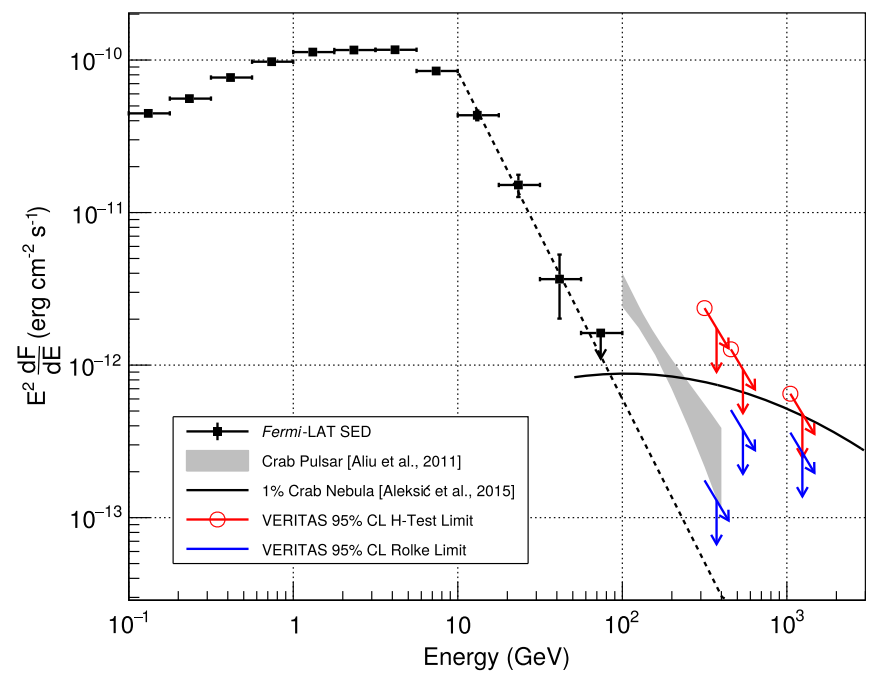

Figure 2. Fermi-LAT spectrum of PSR J0007+7303 (black squares) with VERITAS differential flux upper limits from the soft-, moderate-, and hard-cut analyses. The $H$-Test limits are shown with red arrows, and Rolke limits with blue arrows. A power-law fit to the Fermi data above $10 \mathrm{GeV}$ is given by the black dashed line.

where $\boldsymbol{F}_{0}$ is the flux normalization, $E_{0}$ is fixed to $300 \mathrm{MeV}$, and $\gamma$ is the spectral index fixed to 2 . The normalization of each pulsar is left as a free parameter in the fit.

\subsection{Phase Gating for the VERITAS Analysis}

The regions in the pulsar light curves where signal and background counting are done, also referred to as "phase gates," were defined prior to the application of tests for periodicity. In short, the method utilizes the pulsar light curves presented in the $2 \mathrm{PC}(0.1<E<100 \mathrm{GeV})$ to define the phase gates for the VERITAS search. This method aims to define the gates in such a way as to maximize the detection significance of the VERITAS search by simulating potential VHE light curves that might be obtained in a VERITAS analysis and subsequently finding the optimal phase-gate combination that maximizes the search significance. There are two assumptions invoked in this method: the potential VHE light curve will have the same features as the HE light curve seen in the LAT data (e.g., location and shape of the pulse peaks); and the VHE flux of the pulsar in question is $\sim 1 \%$ of the Crab Nebula flux. If the first assumption is not true, then the search for pulsed emission will be less sensitive. For this reason, we also use the $H$ test to search for pulsed emission.

The method for defining the phase gates for the search for pulsations from the archival pulsars is comprised of the following steps:

1. Determine the signal and background event rates from VERITAS Crab Nebula data using soft cuts. These event rates are obtained in a reflected-region analysis (Fomin et al. 1994).

2. Multiply the rates from step 1 by the source exposure time to get an expected $N_{\text {on }}$ and $\alpha N_{\text {off }}$, then find $N_{\text {excess }}=N_{\text {on }}-\alpha N_{\text {off }}$, where $N_{\text {excess }}$ is the number of excess counts, $N_{\text {on }}$ is the number of counts in the signal region, $N_{\text {off }}$ is the number in the background region, and $\alpha$ is the ratio of the size of the signal region(s) to the background region.
3. Scale the Crab Nebula excess counts found in step 2 by 0.01 to mimic a $1 \%$ Crab Nebula source (the Crab pulsar flux is approximately $1 \%$ of the Crab Nebula flux at $\sim 200 \mathrm{GeV}$ ).

4. Obtain the binned pulse profile from the $2 \mathrm{PC}$ for the pulsar in question and subtract the value of the lowest bin in the profile from all bins to remove the unpulsed component.

5. Normalize the pulse profile from the previous step and multiply each bin by the scaled excess found in step 3 .

6. Add the estimated background expected for the VERITAS observations to the profile by adding $\alpha N_{\text {off }} / N_{\text {bins }}$ to each bin, where $N_{\text {bins }}$ is the total number of bins.

7. Define the number of signal phase gates as one per peak present in the 2PC light curve, and calculate the significance corresponding to all nonoverlapping phasegate bin combinations_-including a background gate. The phase gates selected for the VERITAS analysis are those for which the significance is maximized. As an example, consider a pulsar displaying two HE gamma-ray peaks in its light curve. Two signal phase gates are defined a priori, each with a unique starting and ending edge, which must be located at a bin edge. A background gate is also defined in the same way, and none of these three gates are allowed to overlap. Significances are then calculated for all possible gate combinations by scanning the space of the six possible gate edges. The gate combination with the highest significance is used for the analysis. For cases where two signal phase gates were initially defined but found to be contiguous, the number of gates is reduced to one and the procedure is performed again.

In some cases where a pulsar shows two distinct, nonoverlapping peaks, the significance calculated in this procedure is maximized with only a single signal gate definition. In order to ensure that a signal gate is defined for each peak, a modified three-pass method is used. The first pass is simply the procedure described above. The second pass sets the number of signal phase gates to one and excludes the signal phase gate defined in the first pass from the search region, allowing a signal phase gate to be defined for the peak not found in the first pass. The third pass sets the number of signal phase gates to two, but constrains one of them to be fixed to the signal phase gate determined in pass two. This modified method was necessary to define gates for the light curves of PSR J0205+6449 and PSR J2021+4026. The results of the gating procedure are shown in Figure 1, and phase-gate definitions are given in numerical form in Table 2 .

The latest publicly available timing solutions from the $L A T$ Gamma-ray Pulsar Timing Models webpage are used in the VERITAS analysis; these have a longer epoch of validity than those in the 2PC. The use of these timing solutions to fold the data for the pulsars in most cases introduces appreciable phase offsets with respect to the 2PC light curves, which are calculated and added to the VERITAS event phases, allowing the phase gates derived from the $2 \mathrm{PC}$ light curves to be used in the analysis. To determine the phase offsets for the 13 archival pulsars, a Fermi-LAT data set for each pulsar is phase-folded using both the $2 \mathrm{PC}$ and the latest timing solutions. The resulting light curves are then cross-correlated, and the point where the correlation coefficient is maximized is taken to be the offset in phase. The resulting offset for each pulsar is given in Table 2 . 
(a) PSR J0205+6449

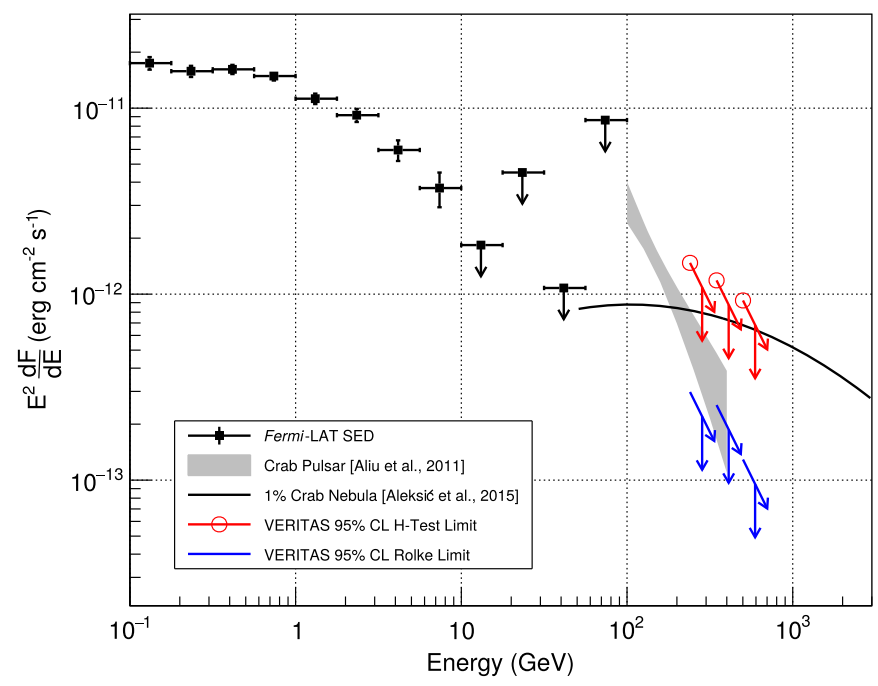

(c) PSR J0357+3205

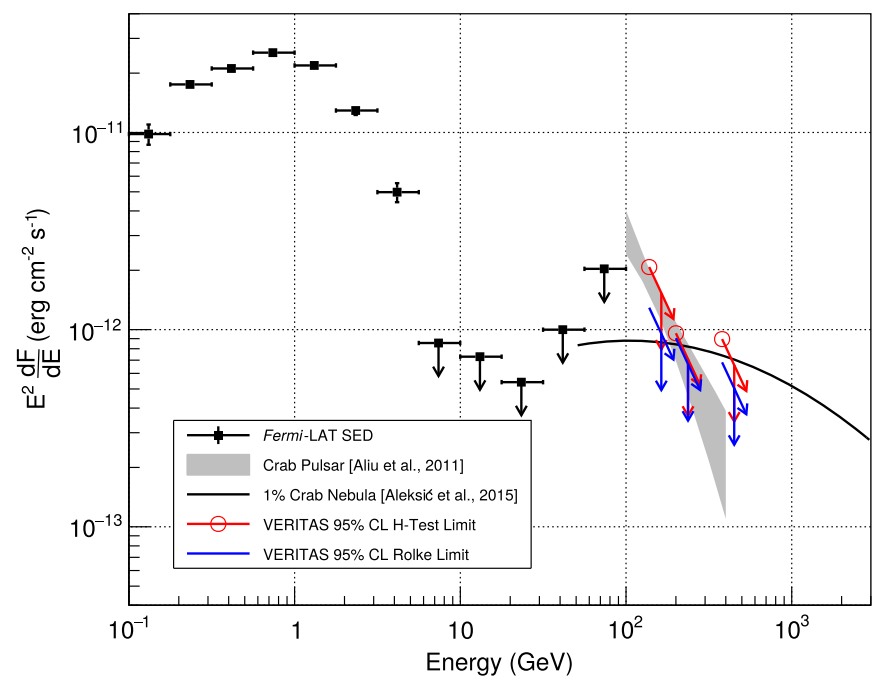

(b) PSR J0248+6021

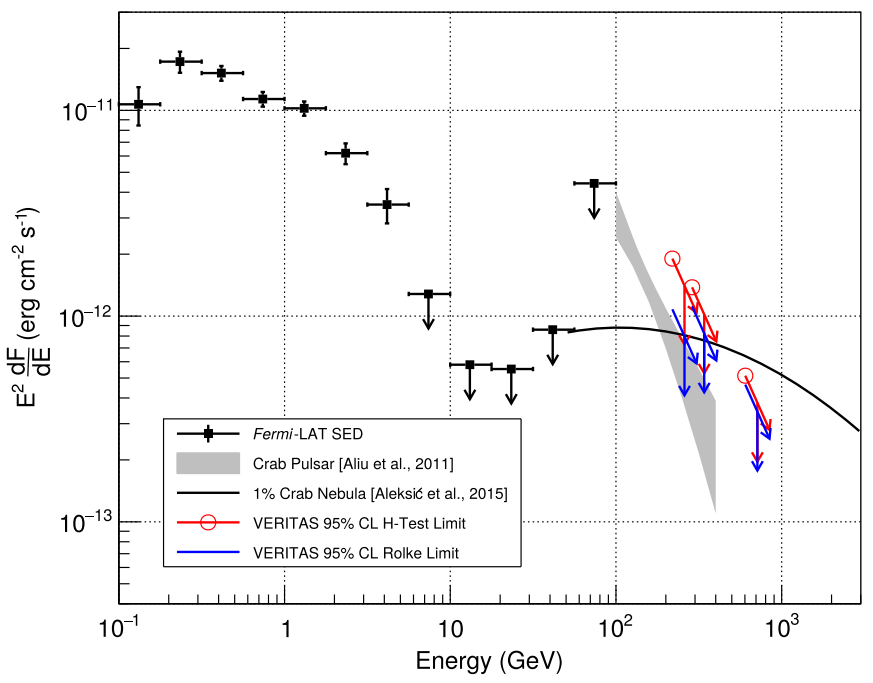

(d) PSR J0631+1036

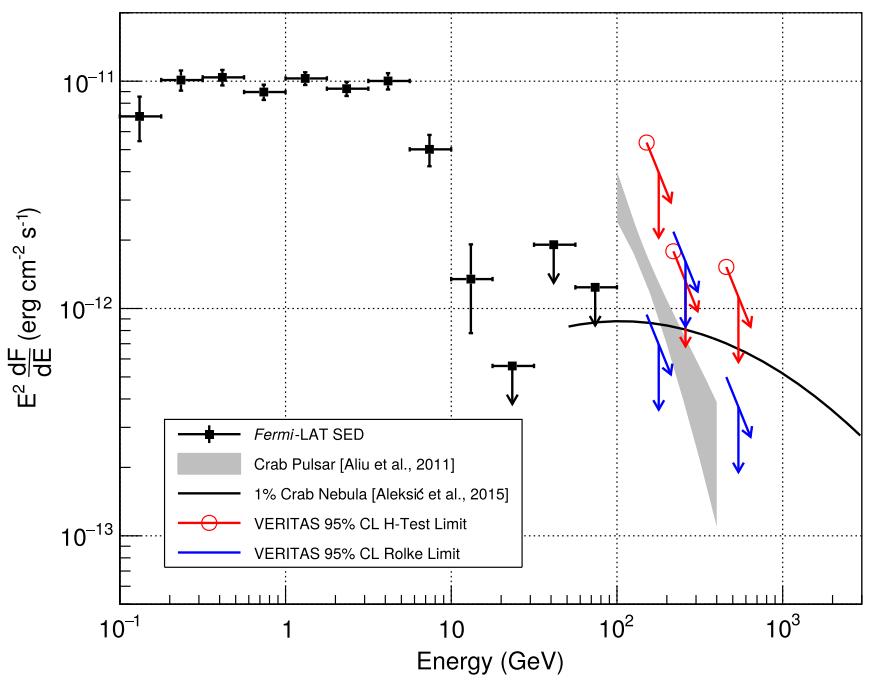

Figure 3. Fermi-LAT spectra (black squares) with VERITAS differential flux upper limits from the soft-, moderate-, and hard-cut analyses. The $H$-Test limits are shown as red arrows, and the Rolke limits as blue arrows. The panels display: PSR J0205+6449 (a), PSR J0248+6021 (b), PSR J0357+3205 (c), and PSR J0631 +1036 (d).

\section{Results}

None of the six periodicity tests applied to each pulsar data set results in the detection of VHE pulsations. The distribution of pre-trials significances from the phase-gate test has a minimum and a maximum value of $-1.93 \sigma$ and $+1.07 \sigma$, respectively. The maximum $H$ statistic is 10.4 , which corresponds to a chance probability of 0.016 . Therefore, none of the tests applied to the data reveal any evidence for pulsed emission in the VERITAS data. Significances and $H$ statistics for each pulsar are given in Table 3.

For each of the six searches for pulsed emission, integral VHE flux upper limits (ULs) from the VERITAS data are computed at the $95 \%$ confidence level. For the phase-gate test results, the Rolke unbounded method (Rolke \& López 2001) is used to set a UL on the excess counts, which is converted into an integral flux UL by dividing by the exposure. For the results from the $H$ test, an integral flux UL is set using the method detailed in de Jager (1994), assuming a single peak with a duty cycle of 10\%. A spectral index of 3.8 is assumed for all UL calculations, which is the same index as seen for the Crab pulsar in Aliu et al. (2011). We note that assuming a significantly harder $(\gamma=2)$ or softer $(\gamma=5)$ spectral index affects the integral flux ULs at a level of $\sim 25 \%$ on average. Spectral analysis energy thresholds for the ULs are taken to be the energy corresponding to the peak of the efficiency ${ }^{31}$ for each analysis. Six 95\% CL integral flux ULs per pulsar are therefore calculated, and are given in Table 3 . We note that the spectral analysis thresholds vary significantly between pulsars, which is primarily due to the different average zenith angle for each set of observations.

The Fermi-LAT spectra derived for each pulsar, along with the VERITAS VHE flux ULs, are presented in Figures 2-5. The Fermi-LAT spectra are all consistent with those reported in

\footnotetext{
${ }^{31}$ Efficiency in this context is the product of the average effective area versus energy curve and the assumed spectrum: $d F / d E \propto E^{-\gamma}$, where here we take $\gamma=3.8$.
} 
(a) PSR J0633+0632

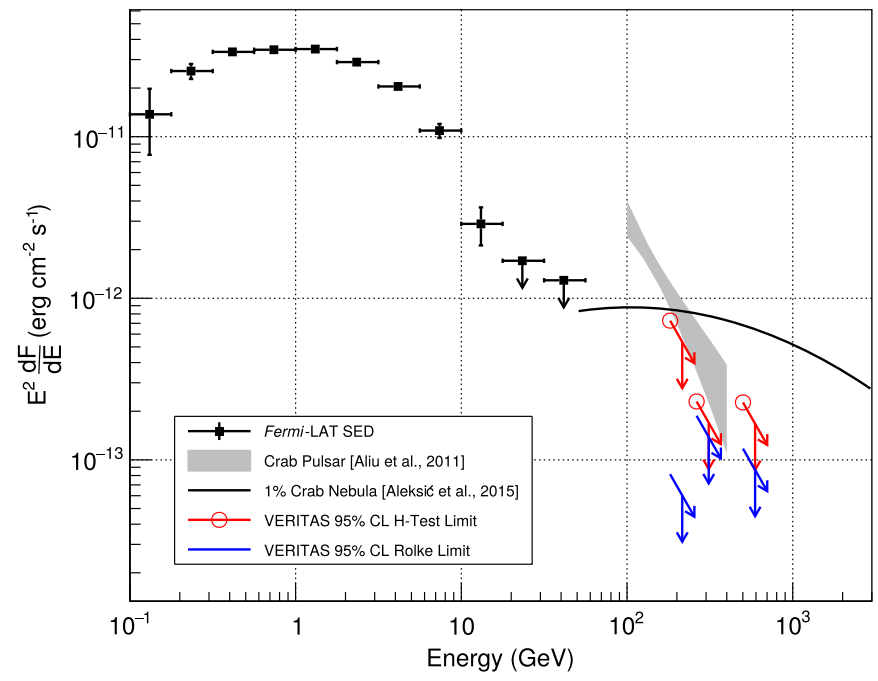

(c) PSR J1954+2836

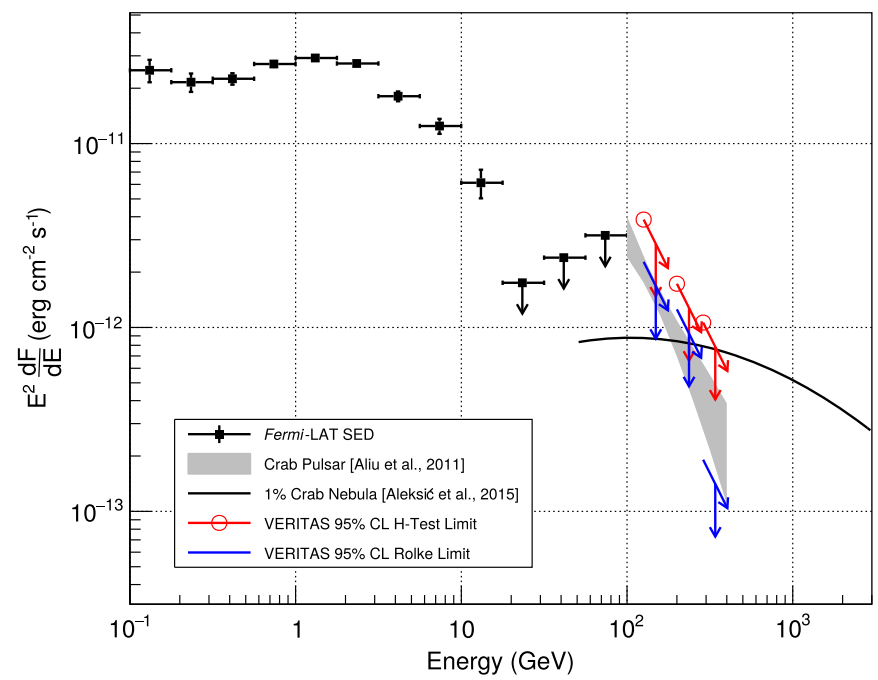

(b) PSR J1907+0602

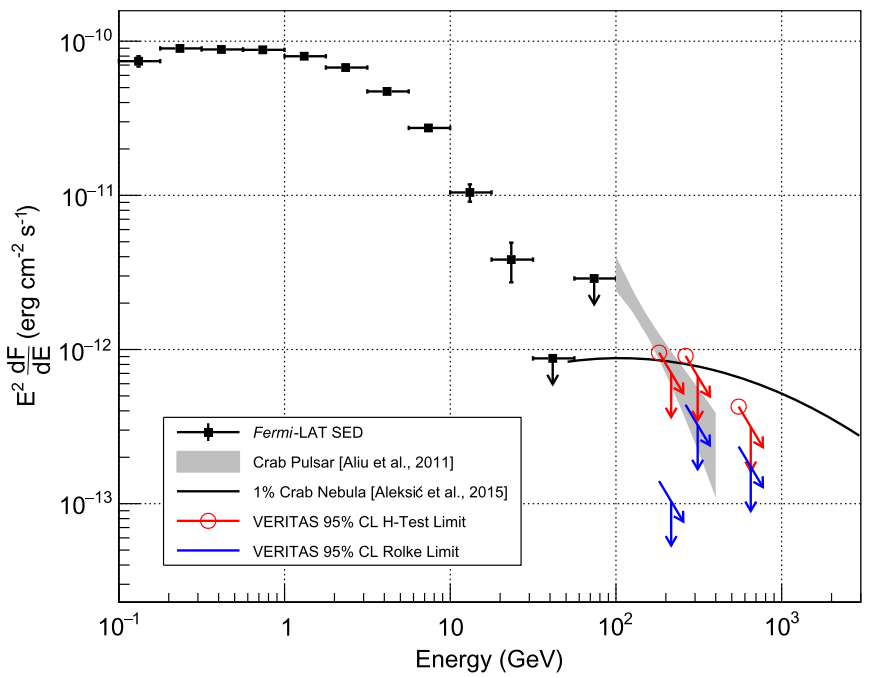

(d) PSR J1958+2846

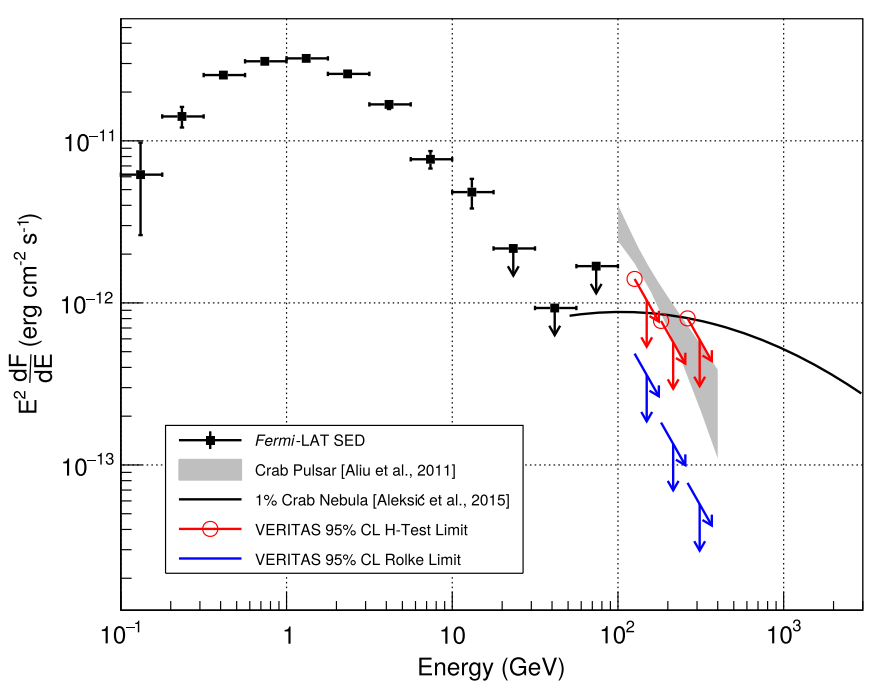

Figure 4. Fermi-LAT spectra (black squares) with VERITAS differential flux upper limits from the soft-, moderate-, and hard-cut analyses. The $H$-Test limits are shown as red arrows, and the Rolke limits as blue arrows. The panels display: PSR J0633+0632 (a), PSR J1907+0602 (b), PSR J1954+2836 (c), and PSR J1958 +2846 (d).

the 2PC (Abdo et al. 2013). For both PSR J0007+7303 and PSR $\mathrm{J} 2021+4026$, sufficient spectral points (at least three) are reconstructed to enable reduced $\chi^{2}$ power-law fits above $10 \mathrm{GeV}$ of the form given in Equation (1), where $E_{0}$ is fixed to $20 \mathrm{GeV}$, and $\gamma$ and $\boldsymbol{F}_{0}$ are left free. The results of the fits are given in Table 4. These fits are intended to help indicate whether or not a power-law extension of the spectrum from HE to VHE is possible, as has been seen for the Crab pulsar. The integral flux ULs given in Table 3 have been converted to differential limits, assuming a spectral index of 3.8, and are plotted at the corresponding spectral analysis threshold. The Fermi spectral points are shown in black. The VERITAS 95\% CL flux ULs from the $H$ test are indicated by the red arrows, and those from the method of Rolke from the phase-gate test are given by the blue arrows. The starting point (nock) of the sloped arrow of each pair is set to the energy threshold and flux UL. These arrows are drawn with a slope to indicate the assumed spectral index of 3.8. For reference, the Crab pulsar spectral bow tie from Aliu et al. (2011) is also shown (gray shaded region), in addition to the Crab Nebula spectral shape from
Aleksić et al. (2015) scaled to $1 \%$ (black curved line). For the two pulsars J0007+7303 and J2021+4026, a reduced- $\chi^{2}$ power-law fit $>10 \mathrm{GeV}$ is given by the black dashed line.

\section{Discussion and Conclusion}

Six searches for pulsed VHE emission from each of 13 pulsars appearing in archival VERITAS data have been performed. No evidence of pulsed VHE emission is found from any pulsar. This search for VHE pulsed emission from the present set of 13 archival pulsars is the first ever done in the VHE band, representing the first comprehensive northern-sky survey of its kind. We note that the ULs constrain a flux that is, in many cases, below the level of the Crab pulsar-so it may be stated broadly that potential pulsed VHE emission from the majority of the pulsars must be fainter than the VHE flux from the Crab pulsar $(\sim 1 \%$ Crab Nebula level). Further, the Fermi-LAT spectral reconstruction did not result in sufficient photon 
(a) PSR J2021+3651

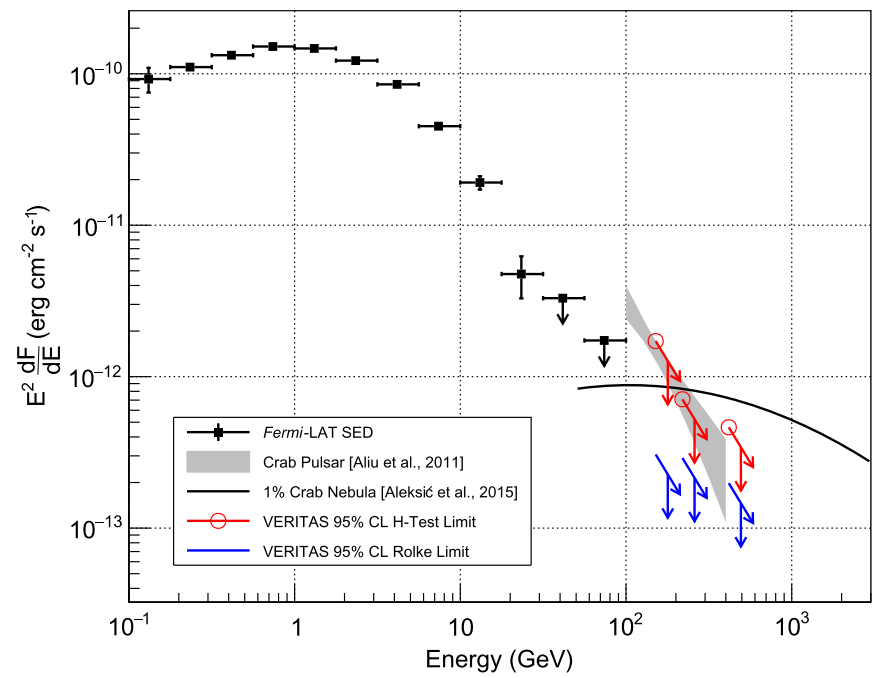

(c) PSR J2032+4127

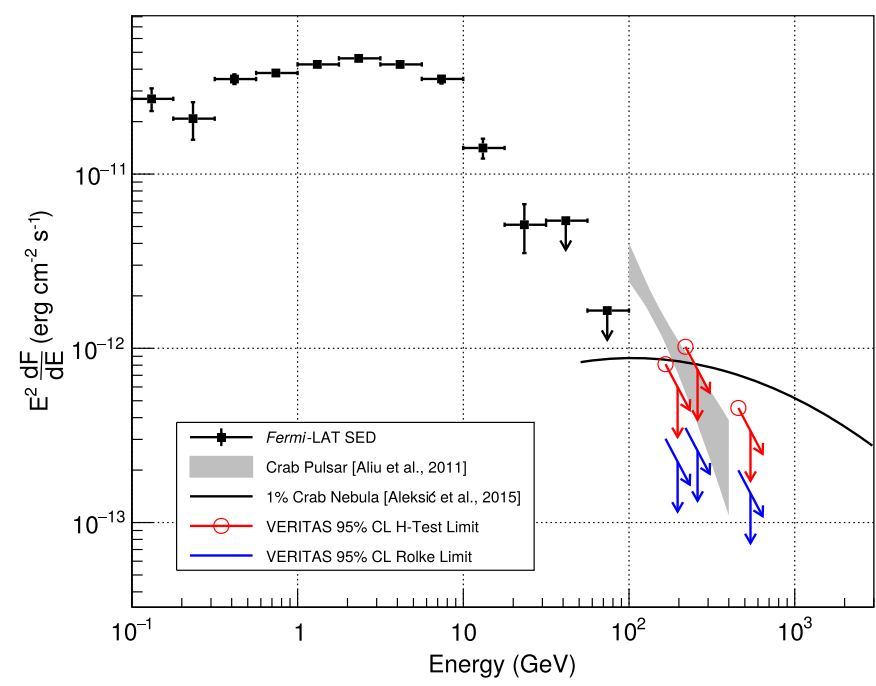

(b) PSR J2021+4026

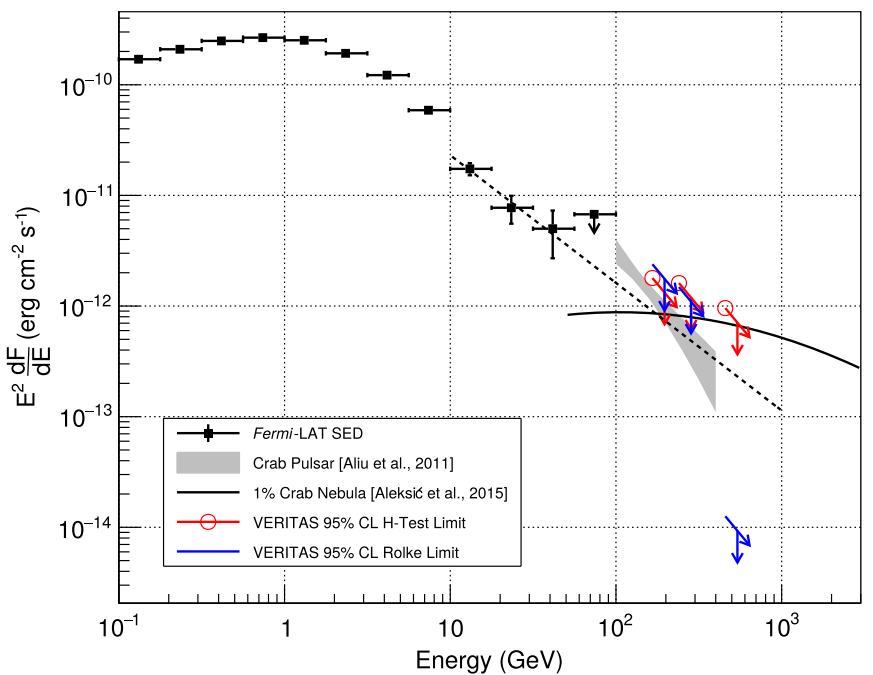

(d) PSR J2229+6114

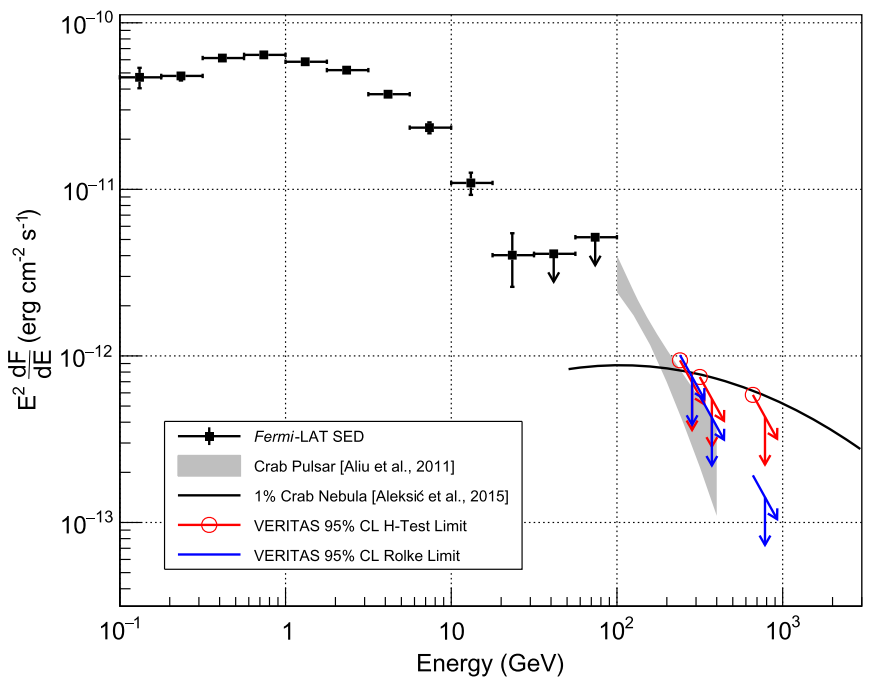

Figure 5. Fermi-LAT spectra (black squares) with VERITAS differential flux upper limits from the soft-, moderate-, and hard-cut analyses. The $H$-Test limits are shown as red arrows, and the Rolke limits as blue arrows. The panels display: PSR J2021+3651 (a), PSR J2021+4026 (b), PSR J2032+4127 (c), and PSR J2229 +6114 (d). A power-law fit to the Fermi data above $10 \mathrm{GeV}$ for PSR J2021+4026 is given by the black dashed line. We note that the highest-energy Rolke flux UL for PSR J2021+4026 appears to constrain a flux level several orders of magnitude below the other ULs; however, this UL corresponds to a large, unphysical negative excess. See Section 4 for some further discussion.

Table 4

Results of the Reduced $\chi^{2}$ Power-law Fits

\begin{tabular}{lcccc}
\hline \hline Pulsar & $\begin{array}{l}\boldsymbol{F}_{0}\left(\times 10^{-7} \mathrm{GeV}^{-1}\right. \\
\left.\mathrm{cm}^{-2} \mathrm{~s}^{-1}\right)\end{array}$ & $\gamma$ & $\chi^{2} /$ n.d.f. & Probability \\
\hline $\mathrm{J} 0007+7303$ & $2.96 \pm 0.29$ & $3.98 \pm 0.24$ & $0.47 / 1$ & 0.49 \\
$\mathrm{~J} 2021+4026$ & $1.60 \pm 0.24$ & $3.23 \pm 0.38$ & $0.25 / 1$ & 0.62 \\
\hline
\end{tabular}

Note. These fits are applied to the Fermi-LAT spectra $>10 \mathrm{GeV}$.

statistics to allow any firm statement about the shapes of the spectra above $10 \mathrm{GeV}$.

The ULs presented here constrain potential spectral hardening or a new spectral component to be at or below the level of the limits. Although pulsar models generally predict a component of VHE emission that is several orders of magnitude below the flux levels probed in this search (e.g., Harding \& Kalapotharakos 2015), another VHE component from the highest-energy particles scattering infrared-to-optical emission may be present at higher energies (Harding et al. 2018). The flux ULs for each pulsar are consistent with synchro-curvature radiation emission scenarios, where the HE gamma-ray spectra are expected to display a power law with an exponential cutoff at a few $\mathrm{GeV}$. One flux UL calculated for PSR J2021+4026 (hard cuts; Rolke method) appears to constrain a possible power-law continuation $>10 \mathrm{GeV}$ into the VHE band. We note that this UL corresponds to the largest down-fluctuation $(-1.9 \sigma)$ in our results - though this low significance is not unexpected, given the total number of tests for signal we perform. ${ }^{32}$ We caution that the possibility of

\footnotetext{
32 That this UL constrains a much lower flux level than the other limits for PSR J2021+4026 is a result of the steep down-fluctuation into a regime where Rolke et al. (2005) caution against overinterpretation of obtained ULs. The method of Feldman \& Cousins (1998) experiences similar difficulty here and gives a UL of zero on the excess counts.
} 


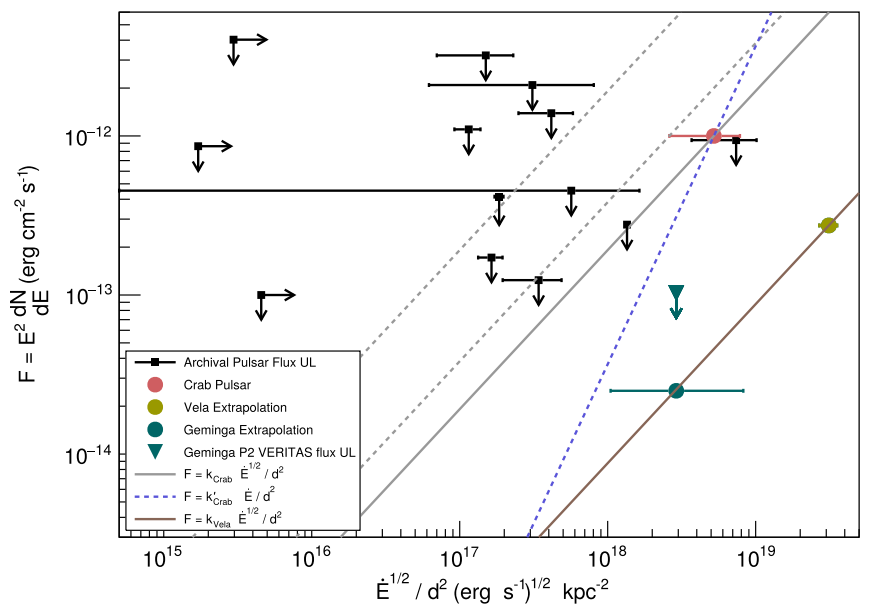

Figure 6. Flux upper limits from the phase-gate test (Rolke method) vs. $\sqrt{\dot{E}} / d^{2}$ for soft cuts. The VHE flux limits are shown by the black squares, and the right-pointing arrows indicate a lower limit on $\sqrt{\dot{E}} / d^{2}$ for pulsars where only a distance limit is available. Error bars come from propagation of the uncertainty on the distance as given in Table 1 . The flux and $\sqrt{\dot{E}} / d^{2}$ for the Crab pulsar are represented by the red dot. Extrapolated fluxes (see text) for the Geminga and Vela pulsars are shown as teal and olive circles, respectively. The Geminga VHE flux upper limit from VERITAS at $135 \mathrm{GeV}$ from Aliu et al. (2015) is shown as a teal triangle and arrow. The Crab pulsar flux shown here is calculated according to the method given in the text. The solid gray line corresponds to $F=k_{\mathrm{Crab}} \sqrt{\dot{E}} / d^{2}$ (see text). The gray dashed lines have the same proportionality, but indicate flux levels of two and ten times the Crab pulsar flux. The blue dashed line corresponds to $F=k_{\mathrm{Crab}}^{\prime} \dot{E} / d^{2}$, which is equivalent to the prediction $L_{\gamma} \propto \dot{E}$ that has been made for the gamma-ray luminosity in some models (e.g., Lyutikov et al. 2012). The solid brown line corresponds to $F=k_{\text {Vela }} \sqrt{\dot{E}} / d^{2}$ (see text).

power-law extensions from the $\mathrm{HE}$ band $>10 \mathrm{GeV}$ to the VHE band is a rather tenuous assumption, given that it is based on what is observed for the only known VHE pulsar-the Crab. The spectral characteristics of other hitherto undetected VHE pulsars may involve significantly different characteristics $>10 \mathrm{GeV}$, such as the presence of the expected exponential cutoff in the HE band in addition to the emergence of a new component at VHEs.

A population study is conducted using the flux ULs derived from the VERITAS data. The VHE flux limits from the phasegate tests for the three sets of cuts are shown as a function of $\sqrt{\dot{E}} / d^{2}$ in Figures 6 and 7. The Crab pulsar flux that is shown for soft cuts is chosen to be $1 \times 10^{-12} \mathrm{erg} \mathrm{cm}^{-2} \mathrm{~s}^{-1}$, which is the approximate flux measured at $200 \mathrm{GeV}$ by VERITAS (Aliu et al. 2011). For moderate and hard cuts, the Crab flux is extrapolated to 300 and $500 \mathrm{GeV}$, respectively, according to a power law with $\Gamma=3.8$ (the same spectral index for the Crab pulsar measured in Aliu et al. (2011). These energies are chosen to approximately match the average energy thresholds given in Table 3.

An assumption that the flux $F$ is proportional to $\sqrt{\dot{E}} / d^{2}$ (gray and brown lines in Figures 6 and 7) is a restatement of the equivalent assumption $L_{\gamma} \propto \sqrt{\dot{E}}$, where $L_{\gamma}$ is the gamma-ray luminosity, because $F \propto L_{\gamma} / d^{2}$. The prediction that $L_{\gamma} \propto \sqrt{\dot{E}}$ is expected in models that assume a linear dependence of $L_{\gamma}$ on the open-field-line voltage in the gap (Arons 1996). The 2PC FermiLAT gamma-ray pulsar population plotted as $L_{\gamma}$ against $\dot{E}$ roughly follows a power-law trend (Abdo et al. 2013), though there is likely too much scatter in the data to make a firm empirical claim about the proportionality of $L_{\gamma}$ on $\sqrt{\dot{E}}$ in HE gamma-rays.
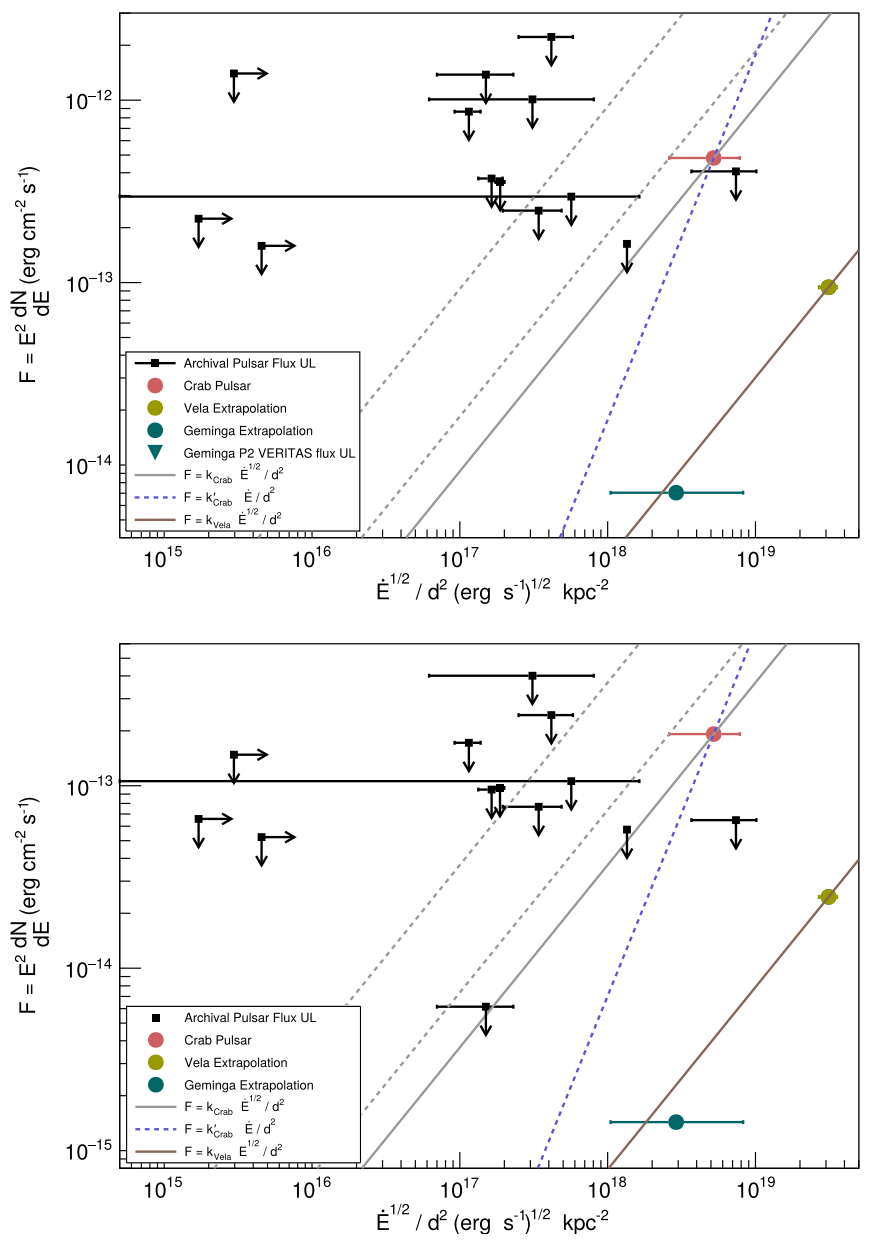

Figure 7. Same as Figure 6, but for moderate cuts (top) and hard cuts (bottom). See Figure 6 caption for details.

Adopting the assumption that young gamma-ray pulsars should all have a flux $F=k \sqrt{\dot{E}} / d^{2}$, where $k$ is a constant of proportionality, we can set $k$ based on what is known for the Crab pulsar:

$$
k_{\mathrm{Crab}}=F_{\mathrm{Crab}} d_{\mathrm{Crab}}^{2} / \sqrt{\dot{E}_{\mathrm{Crab}}} .
$$

If true, this assumption would result in VHE pulsar fluxes trending around the solid gray line corresponding to $F=k_{\text {Crab }} \sqrt{\dot{E}} / d^{2}$ in Figures 6 and 7. Almost all of the VERITAS flux ULs lie well above this line, so this prediction for the fluxes of VHE pulsars remains unconstrained in most cases. However, the three flux limits for one pulsar (PSR J2229 $+6114)$ all fall below the gray line. The error on $\sqrt{\dot{E}} / d^{2}$ due to the uncertainty on the distance measurement places the PSR $\mathrm{J} 2229+6114$ limits within $1 \sigma$ (soft and moderate cuts) or $2 \sigma$ (hard cuts) of the gray line, so no firm claim regarding the validity of the trend can be made with the UL for this pulsar. Furthermore, systematic errors on distance measurements are typically quite large. VHE flux estimates for the Vela and Geminga pulsars are derived via power-law extrapolation of their Fermi-LAT spectra above $10 \mathrm{GeV}$ to 200,300 , and $500 \mathrm{GeV}$ for soft, moderate, and hard cuts, respectively. These flux estimates are shown in Figures 6 and 7, and lie well below the $F=k \sqrt{\dot{E}} / d^{2}$ expectation for $k=k_{\text {Crab }}$. Measures of $\dot{E}$ and 
the distances for Geminga and Vela are taken from the 2PC (Abdo et al. 2013, references therein for the distances are Verbiest et al. 2012 and Dodson et al. 2003, respectively). That these flux extrapolations lie well below the gray lines in Figures 6 and 7 challenges the assumption that the fluxes of VHE pulsars may follow the assumed trend. If instead the assumption $k=k_{\text {Vela }}$ is made, such that $F=k_{\text {Vela }} \sqrt{\dot{E}} / d^{2}$, the VHE fluxes should trend around the brown lines in Figures 6 and 7 . We note that, for all three sets of cuts, the brown line intersects the extrapolated Geminga flux point error. It could be the case that other VHE pulsars follow this assumed $\sqrt{\dot{E}}$ trend, though with a much lower value for $k$ than $k_{\text {Crab }}$.

The upcoming Cherenkov Telescope Array (CTA) will boast a significantly reduced low-energy threshold and significantly improved sensitivity over current-generation instruments. Observations with CTA will therefore be able to obtain far more constraining flux ULs for the same exposure time (Cherenkov Telescope Array Consortium 2019), and the results presented here can help guide future observations with CTA. The firm detection of more pulsars above $100 \mathrm{GeV}$ remains an important scientific endeavor, given that the nature of their VHE emission mostly remains unresolved for now.
This research is supported by grants from the U.S. Department of Energy Office of Science, the U.S. National Science Foundation, the Smithsonian Institution, and Canada's NSERC. We acknowledge the excellent work of the technical support staff at the Fred Lawrence Whipple Observatory, as well as at the collaborating institutions, in the construction and operation of the instrument.

Software: Tempo2 (Hobbs et al. 2006), Fermi-LAT Science Tools (v10r0p5), ROOT (5.34.36).

\section{Appendix}

\section{A1. Pulse Profiles from the VERITAS Data}

The pulsar light curves obtained by phase folding the VERITAS data for each of the 13 archival pulsars are shown in Figure Set 8 . The complete figure set (39 images) is available in the online journal. Each figure shows the P1 and P2 (where applicable) phase gates from Table 2 in green, with the background gate shown in gray. The red dashed line indicates the average background counts, with the two red dotted lines giving the $\pm 1 \sigma$ deviation. The inset text box gives $N_{\text {on }}, \alpha N_{\text {off }}$, $N_{\text {excess }}$, and the significance from Equation (17) in $\mathrm{Li} \& \mathrm{Ma}$ (1983), in that order from top to bottom. 

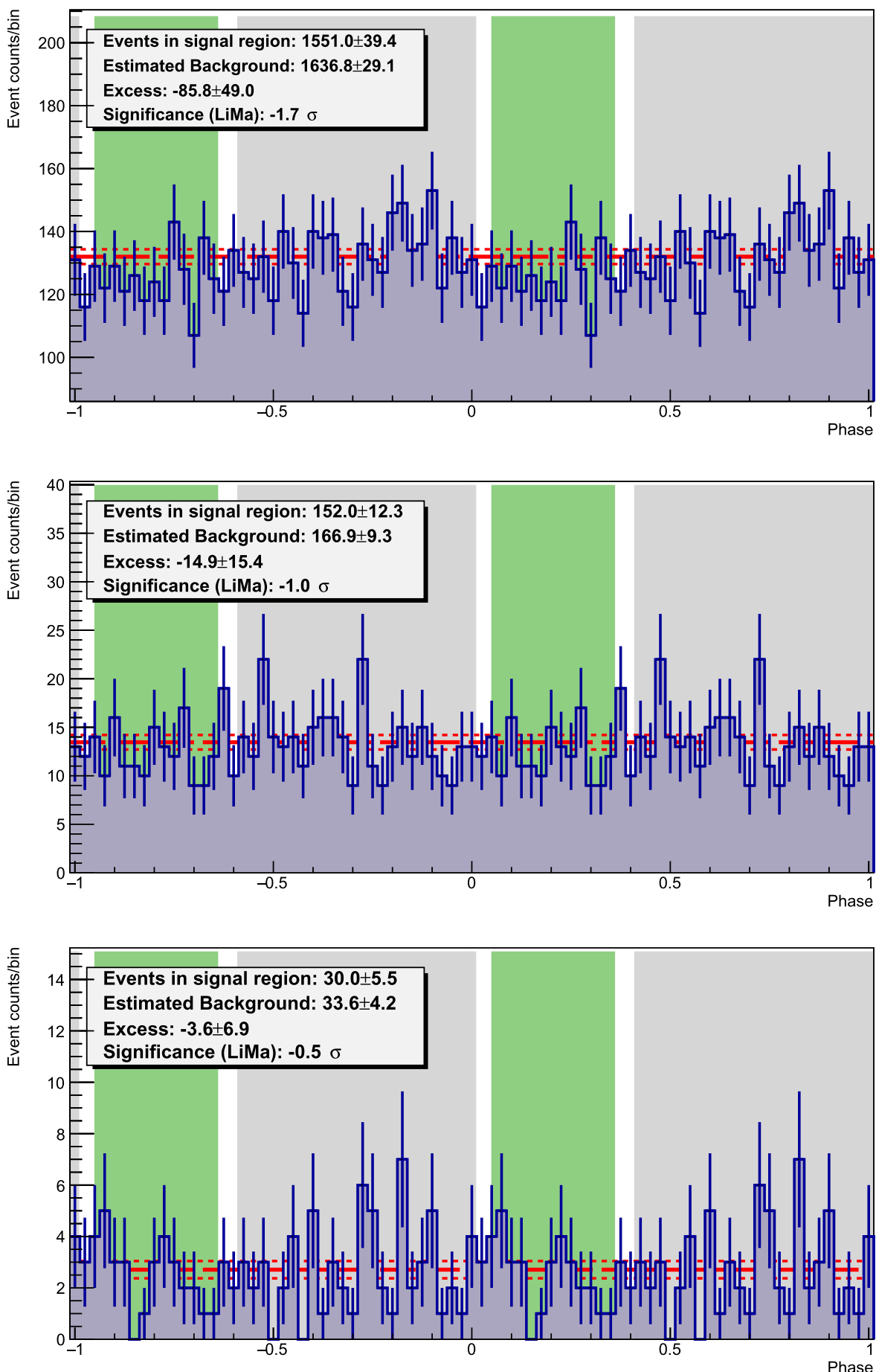

Figure 8. Pulse profiles of PSR J0007+7303 from VERITAS data for soft cuts (top panel), moderate cuts (middle panel), and hard cuts (bottom panel). The pulse profiles of all 13 pulsars are available in the figure set.

(The complete figure set (39 images) is available.)

\section{ORCID iDs}

W. Benbow (i) https://orcid.org/0000-0003-2098-170X

R. Bird (10 https://orcid.org/0000-0002-4596-8563

W. Cui (1) https://orcid.org/0000-0002-6324-5772

Q. Feng (i) https://orcid.org/0000-0001-6674-4238

L. Fortson (i) https://orcid.org/0000-0002-1067-8558

A. Furniss (ib https://orcid.org/0000-0003-1614-1273

D. Hanna (1) https://orcid.org/0000-0002-8513-5603
O. Hervet (ib https://orcid.org/0000-0003-3878-1677

C. A. Johnson (1) https://orcid.org/0000-0002-0641-7320

P. Kaaret (1) https://orcid.org/0000-0002-3638-0637

D. Kieda (1) https://orcid.org/0000-0003-4785-0101

R. Mukherjee (1) https://orcid.org/0000-0002-3223-0754

A. N. Otte (i) https://orcid.org/0000-0002-5955-6383

M. Pohl (1) https://orcid.org/0000-0001-7861-1707

E. Pueschel (1) https://orcid.org/0000-0002-0529-1973 
G. T. Richards (ib https://orcid.org/0000-0002-1408-807X

M. Santander (10 https://orcid.org/0000-0001-7297-8217

K. Shahinyan (1) https://orcid.org/0000-0001-5128-4160

I. Sushch (1) https://orcid.org/0000-0002-2814-1257

\section{References}

Abdalla, H., Aharonian, F., Ait Benkhali, F., et al. 2018, A\&A, 620, A66 Abdo, A. A., Ackermann, M., Ajello, M., et al. 2009, Sci, 325, 840 Abdo, A. A., Ackermann, M., Ajello, M., et al. 2010, ApJ, 711, 64 Abdo, A. A., Ajello, M., Allafort, A., et al. 2013, ApJS, 208, 17

Abeysekara, A. U., Benbow, W., Bird, R., et al. 2018, ApJL, 867, L19 Acciari, V. A., Beilicke, M., Blaylock, G., et al. 2008, ApJ, 679, 1427 Acero, F., Ackermann, M., Ajello, M., et al. 2015, ApJS, 218, 23 Ackermann, M., Ajello, M., Albert, A., et al. 2012, ApJS, 203, 4 Ackermann, M., Ajello, M., Atwood, W. B., et al. 2016, ApJS, 222, 5 Aharonian, F. A., Bogovalov, S. V., \& Khangulyan, D. 2012, Natur, 482, 507 Ahnen, M. L., Ansoldi, S., Antonelli, L. A., et al. 2016, A\&A, 591, A138 Albert, J., Aliu, E., Anderhub, H., et al. 2008, ApJL, 675, L25 Aleksić, J., Alvarez, E. A., Antonelli, L. A., et al. 2012, A\&A, 540, A69 Aleksić, J., Ansoldi, S., Antonelli, L. A., et al. 2014, A\&A, 567, L8 Aleksić, J., Ansoldi, S., Antonelli, L. A., et al. 2015, JHEAp, 5, 30 Aliu, E., Archambault, S., Archer, A., et al. 2015, ApJ, 800, 61 Aliu, E., Archambault, S., Arlen, T., et al. 2013a, ApJ, 764, 38 Aliu, E., Archambault, S., Arlen, T., et al. 2013b, ApJ, 770, 93

Aliu, E., Arlen, T., Aune, T., et al. 2011, Sci, 334, 69

Aliu, E., Aune, T., Behera, B., et al. 2014, ApJ, 783, 16

Allafort, A., Baldini, L., Ballet, J., et al. 2013, ApJL, 777, L2

Ansoldi, S., Antonelli, L. A., Antoranz, P., et al. 2016, A\&A, 585, A133

Arons, J. 1996, A\&AS, 120, 49

Atwood, W. B., Abdo, A. A., Ackermann, M., et al. 2009, ApJ, 697, 1071

Caraveo, P. A. 2014, ARA\&A, 52, 211

Cherenkov Telescope Array Consortium 2019, Science with the Cherenkov Telescope Array (Singapore: World Scientific)

de Jager, O. C. 1994, ApJ, 436, 239

de Jager, O. C., Raubenheimer, B. C., \& Swanepoel, J. W. H. 1989, A\&A, 221,180

De Luca, A., Marelli, M., Mignani, R. P., et al. 2011, ApJ, 733, 104

Dodson, R., Legge, D., Reynolds, J. E., \& McCulloch, P. M. 2003, ApJ, 596, 1137

Feldman, G. J., \& Cousins, R. D. 1998, PhRvD, 57, 3873

Fomin, V. P., Stepanian, A. A., Lamb, R. C., et al. 1994, APh, 2, 137
Gaia Collaboration, Brown, A. G. A., Vallenari, A., et al. 2018, A\&A, 616, A1

Halpern, J. P., Gotthelf, E. V., Camilo, F., Helfand, D. J., \& Ransom, S. M. 2004, ApJ, 612, 398

Harding, A. K., \& Kalapotharakos, C. 2015, ApJ, 811, 63

Harding, A. K., Kalapotharakos, C., Barnard, M., \& Venter, C. 2018, ApJL, 869, L18

Ho, W. C. G., Ng, C.-Y., Lyne, A. G., et al. 2017, MNRAS, 464, 1211

Hobbs, G. B., Edwards, R. T., \& Manchester, R. N. 2006, MNRAS, 369, 655

Hohle, M. M., Neuhäuser, R., \& Schutz, B. F. 2010, AN, 331, 349

Kirichenko, A., Danilenko, A., Shternin, P., et al. 2015, ApJ, 802, 17

Kothes, R., Uyaniker, B., \& Pineault, S. 2001, ApJ, 560, 236

Ladouceur, Y., \& Pineault, S. 2008, A\&A, 490, 197

Landecker, T. L., Roger, R. S., \& Higgs, L. A. 1980, A\&AS, 39, 133

Leung, G. C. K., Takata, J., Ng, C. W., et al. 2014, ApJL, 797, L13

Li, J., Torres, D. F., de Oña Wilhelmi, E., Rea, N., \& Martin, J. 2016, ApJ, 831,19

Li, T.-P., \& Ma, Y.-Q. 1983, ApJ, 272, 317

Lyne, A., \& Graham-Smith, F. 2012, Pulsar Astronomy (Cambridge: Cambridge Univ. Press)

Lyne, A. G., Stappers, B. W., Keith, M. J., et al. 2015, MNRAS, 451, 581

Lyutikov, M., Otte, N., \& McCann, A. 2012, ApJ, 754, 33

Manchester, R. N., Hobbs, G. B., Teoh, A., \& Hobbs, M. 2005, AJ, 129, 1993

Marelli, M., De Luca, A., Salvetti, D., et al. 2013, ApJ, 765, 36

McCann, A. 2015, ApJ, 804, 86

Mirzoyan, R., \& Mukherjee, R. 2017, ATel, 10971

Mochol, I., \& Pétri, J. 2015, MNRAS, 449, L51

Park, N. \& VERITAS Collaboration 2015, ICRC, 34, 771

Pierbattista, M., Harding, A. K., Grenier, I. A., et al. 2015, A\&A, 575, A3

Pineault, S., Landecker, T. L., Madore, B., \& Gaumont-Guay, S. 1993, AJ, 105,1060

Rolke, W. A., \& López, A. M. 2001, NIMPA, 458, 745

Rolke, W. A., López, A. M., \& Conrad, J. 2005, NIMPA, 551, 493

Theureau, G., Parent, D., Cognard, I., et al. 2011, A\&A, 525, A94

Verbiest, J. P. W., Weisberg, J. M., Chael, A. A., Lee, K. J., \& Lorimer, D. R. 2012, ApJ, 755, 39

Watters, K. P., Romani, R. W., Weltevrede, P., \& Johnston, S. 2009, ApJ, 695, 1289

Xu, Y., Reid, M. J., Zheng, X. W., \& Menten, K. M. 2006, Sci, 311, 54

Zanin, R. 2017, EPJWC, 136, 03003

Zepka, A., Cordes, J. M., Wasserman, I., \& Lundgren, S. C. 1996, ApJ, 456, 305

Zhao, J., Ng, C. W., Lin, L. C. C., et al. 2017, ApJ, 842, 53 